\title{
Regeneração natural em Mata de Restinga em área de pecuária extensiva no Município de Pelotas, extremo Sul do Brasil
}

\author{
Tarso Vieira da Costa ${ }^{1}$, Tiago Schuch Lemos Venzke ${ }^{2 *}$ \\ 1 Universidade Católica de Pelotas, Rua Félix da Cunha, 412, CEP 96010-000, Pelotas, RS, Brasil \\ ${ }^{2}$ Universidade Federal de Pelotas, Campus Universitário, s/n, C P 354, CEP 96010-900, Pelotas, RS, Brasil
}

^Autor correspondente:
venzke.tiago@gmail.com

Termos para indexação:

Conservação de recursos

Restauração ecológica

Área degradada

Index terms:

Resource conservation

Ecological restoration

Degraded land

Histórico do artigo:

Recebido em 08/09/2015

Aprovado em 16/09/2016

Publicado em 30/12/2016

doi: $10.4336 / 2016 . p f b .36 .88 .922$

\begin{abstract}
Resumo - Foi analisada a regeneração natural em mata de restinga ocupada por pecuária extensiva no Município de Pelotas, RS. Foram delimitadas duas áreas, uma com acesso do gado ao interior da floresta e outra sem acesso (controle). Foram utilizados dois transectos com 20 parcelas sistemáticas de $2 \mathrm{~m}$ x $2 \mathrm{~m}$ distantes $5 \mathrm{~m}$. Foram incluídos indivíduos arbóreo-arbustivos com no mínimo $50 \mathrm{~cm}$ de altura e no máximo $5 \mathrm{~cm}$ de DAS (diâmetro à altura do solo). Foram coletadas 27 espécies em 14 famílias. Na área controle foram encontradas 23 espécies e 316 indivíduos, enquanto na área com pecuária foram amostradas 10 espécies e 36 indivíduos. As espécies com maior abundância na área controle foram Psychotria carthagenensis Jacq. (91 ind.), Sorocea bonplandii (Baill.) W.C.Burger et al. (82), P. brachyceras Müll.Arg. (62), Trichilia elegans A.Juss. (15) e Eugenia uruguayensis Cambess. (11). Na área com pecuária, as mais abundantes foram Daphnopsis racemosa Griseb. (17 ind.), P. brachyceras (6), Solanum mauritianum Scop. (5), P. carthagenensis (2) e T. elegans (1). Os resultados mostraram diferença significativa da regeneração natural entre os dois trechos amostrados. Esses resultados corroboram com a hipótese de que a atividade pecuária influencia negativamente a riqueza e a estrutura da regeneração natural de comunidades florestais.
\end{abstract}

\section{Natural regeneration in Restinga forest under extensive cattle ranching in municipality of Pelotas, extreme South of Brazil}

\begin{abstract}
Natural regeneration was analyzed on Restinga Forest, located in extensive cattle ranching area in the Municipality of Pelotas, Rio Grande do Sul State, Brazil. Two areas were delimited, one with cattle access and the other without (control). Two transects with 20 systematic plots of $2 \mathrm{~m} \times 2 \mathrm{~m}$ distant $5 \mathrm{~m}$ were used. We collected 27 species from 14 families. In control area 23 species and 316 individuals were registered, while in the area with cattle only 10 species and 36 individuals were sampled. The species with the highest abundance in control area were Psychotria carthagenensis Jacq. (91 ind.), Sorocea bonplandii (Baill.) W.C.Burger et al. (82), P. brachyceras Müll.Arg. (62), Trichilia elegans A.Juss. (15) and Eugenia uruguayensis Cambess. (11). The area with cattle access were Daphnopsis racemosa Griseb. (17 ind.), P. brachyceras (6), Solanum mauritianum Scop. (5), P. carthagenensis (2) and T. elegans (1). The results showed significant differences in natural regeneration, corroborating the hypothesis that livestock activities influence negatively the richness and the structure of natural regeneration of forest communities.
\end{abstract}




\section{Introdução}

A regeneração natural é o mecanismo de renovação da floresta, decorrendo da interação de processos naturais de restabelecimento do ecossistema florestal e, portanto, parte do ciclo de crescimento da floresta (Gama et al., 2002). Nesse estágio, a limitação no recrutamento é um dos mecanismos mais importantes que controlam a regeneração florestal (Carvalho, 1984; Alves \& Metzger, 2006).

O entendimento da regeneração natural serve para compreender a dinâmica da vegetação, permitindo realizar previsões sobre comportamento e desenvolvimento futuro das florestas, bem como informações para o silvicultor poder favorecer o crescimento e maximizar o volume de espécies desejáveis (Gama et al., 2002). Magnago et al. (2012) salientam que a regeneração natural é ferramenta para a restauração ecológica de florestas, principalmente para grandes áreas geográficas, onde os métodos de plantio podem ser inviáveis, devido aos elevados custos financeiros nas fases de implantação e manutenção dos projetos de reflorestamento.

Frente a isso, autores mostram a ocorrência de alterações da interação da pecuária sobre a vegetação e a regeneração natural (Szaro \& Pase, 1983; Schulz \& Leininger, 1990; Stern et al., 2002; Vargas et al., 2002; Tobler et al., 2003; Lucas et al., 2004; Sosinski Júnior \& Pillar, 2004; Sampaio \& Guarino, 2007; Santos \& Souza, 2007; Meers et al., 2008; Smale et al., 2008; Cândido \& Silva, 2010; Rosolen et al., 2012; Castilhos \& Pillar, 2014). Assim, a análise da regeneração natural em áreas com presença de gado é importante para gerar informações para gestão ambiental e planos de manejo com abordagens legais, práticas e conservacionistas.

O objetivo desse trabalho foi avaliar a regeneração natural de espécies arbustivo-arbóreas em um remanescente de Mata de Restinga em área de sistema de pecuária extensiva no Município de Pelotas, RS.

\section{Material e métodos}

A área de estudo se encontra na "Mata da Praia do Totó" (31'43'25”'S e 52 11 '46”'W, altitude < $10 \mathrm{~m}$ ), localizada na Praia do Laranjal, Município de Pelotas, Planície Costeira do Rio Grande do sul. De acordo com a classificação do IBGE para vegetação brasileira, trata-se de Área de Formação Pioneira com Influência Fluvial (IBGE, 1986), sendo denominada como Mata de
Restinga Arenosa (Waechter, 1990). A mata se localiza dentro das condições dinâmicas do estuário da Laguna dos Patos e com uma distância de aproximadamente 48 $\mathrm{km}$ do oceano (Venzke et al., 2012).

O remanescente florestal possui cerca de 6.200 $\mathrm{m}$ de comprimento, largura média de $266 \mathrm{~m}$ e aproximadamente 160 ha (Universidade Católica de Pelotas, 2006), cuja configuração alongada estendese paralela à margem da Laguna dos Patos. O local é protegido ambientalmente pela legislação municipal que declarou a Mata da Praia do Totó como área de valor paisagístico e ecológico, localizada no Balneário do Laranjal e do Barro Duro (Pelotas, 1998).

O clima do local na classificação climática do sistema de Köppen é subtropical úmido ou do tipo "cfa". A temperatura média anual na região é de $17,8{ }^{\circ} \mathrm{C}$ com médias mensais de $23,2{ }^{\circ} \mathrm{C}$ para o mês mais quente (janeiro) e $12,4{ }^{\circ} \mathrm{C}$ para o mês mais frio (julho). A precipitação pluviométrica média anual é $1.367 \mathrm{~mm}$ e normalmente não ocorrem meses com déficit hídrico. As precipitações menores ocorrem em março $(97,4 \mathrm{~mm})$ e em novembro $(99,5 \mathrm{~mm})$; as maiores em fevereiro e em julho, com 153,3 mm e $146 \mathrm{~mm}$, respectivamente (Embrapa Clima Temperado, 2011).

Foram analisados dois blocos amostrais distribuídos em dois transectos equidistantes $5 \mathrm{~m}$ e separados por uma cerca de cinco fios de arame. Um transecto com presença de pastoreio e o outro sem pecuária e servindo como área controle. Os dois blocos amostrais tinham características de Mata de Restinga Arenosa como drenagem eficiente e sem condições de encharcamento edáfico.

Para cada transecto foram coletadas 20 parcelas sistemáticas de $2 \mathrm{~m} \mathrm{x} 2 \mathrm{~m}$, totalizando $80 \mathrm{~m}^{2}$. O critério de inclusão dos indivíduos foi no mínimo de $50 \mathrm{~cm}$ de altura e no máximo $5 \mathrm{~cm}$ de DAS (diâmetro à altura do solo). A medida de DAS foi realizada no caule a $3 \mathrm{~cm}$ do solo.

Para análise da estrutura da regeneração natural foram avaliados os parâmetros fitossociológicos de densidade, frequência e dominância absolutos e relativos e valor de importância (Mueller-Dombois \& Ellenberg, 1974). Esses parâmetros fitossociológicos foram calculados para cada área pelo programa "Fitopac 2" (Shepherd, 1995), assim como os índices de diversidade de Shannon-Wiener (H') e a equabilidade de Pielou (J'). Para avaliação das diferenças entre tratamentos, utilizouse o teste t, com nível de significância de $95 \%$, após atender os pressupostos de normalidade. As análises foram realizadas no programa PAST. 


\section{Resultados e discussão}

O levantamento da regeneração natural resultou num total de 27 espécies, 21 gêneros e 14 famílias (Tabela 1). Foram amostradas 23 espécies e 11 famílias na área controle e 10 espécies e 8 famílias na área com presença do gado. As famílias com maior número de espécies foram Myrtaceae (7), seguida por Rubiaceae (3) e Salicaceae, Euphorbiaceae, Sapindaceae, Lauraceae e Meliaceae com duas espécies cada. As famílias com maior abundância de indivíduos na área com gado foram Thymelaeaceae (17), Rubiaceae (9) e Solanaceae (5) e na área controle Rubiaceae (163), Moraceae (82), Meliaceae (20), Myrtaceae (18) e Euphorbiaceae (10).

Indivíduos da família Myrtaceae não foram coletados na área com gado, enquanto na área sem gado esta família foi a quarta em número de indivíduos. Scherer et al. (2007) encontrou Myrtaceae como a família melhor representada na regeneração natural e no extrato juvenil, com mais de $60 \%$ de potencial da regeneração natural em Mata de Restinga Arenosa no Parque Estadual do Itapuã, estado do Rio Grande do Sul. Myrtaceae já tinha apresentado o maior número de espécies na florística da Mata do Totó (Venzke et al., 2012) e em diferentes fragmentos florestais no município de Pelotas (Venzke, 2012). Em geral, Myrtaceae na flora da Planície Costeira do Rio Grande do Sul, tem elevada riqueza e importância na estrutura do componente arbóreo de Matas de Restinga Arenosas (Dillenburg et al., 1992; Waechter et al., 2000; Moraes \& Mondin, 2001; Dorneles \& Waechter, 2004; Scherer et al., 2005).

Tabela 1. Lista das espécies amostradas na regeneração natural em uma Mata de Restinga Arenosa, no extremo sul do Brasil.

\begin{tabular}{lcc}
\hline \multicolumn{1}{c}{ Famílias/Espécie } & $\mathrm{s} / \mathrm{g}$ & $\mathrm{c} / \mathrm{g}$ \\
\hline $\begin{array}{l}\text { Aquifoliaceae } \\
\text { Ilex dumosa Reissek }\end{array}$ & - & $\mathrm{x}$ \\
$\begin{array}{l}\text { Erythroxylaceae } \\
\text { Erythroxylum argentinum } \text { O. E. Schulz }\end{array}$ & - & $\mathrm{x}$ \\
$\begin{array}{l}\text { Euphorbiaceae } \\
\text { Sebastiania brasiliensis Spreng. }\end{array}$ & & \\
$\begin{array}{l}\text { Sebastiania commersoniana } \text { (Baill.) L. B. Sm. \& R. J. } \\
\text { Downs }\end{array}$ & $\mathrm{x}$ & $\mathrm{x}$ \\
Lauraceae & & - \\
Nectandra megapotamica (Spreng.) Mez & $\mathrm{x}$ & - \\
$\begin{array}{l}\text { Ocotea pulchella } \text { (Nees \& Mart.) Mez } \\
\text { Meliaceae }\end{array}$ & $\mathrm{x}$ & - \\
Trichilia clausseni C. DC. & & \\
Trichilia elegans A. Juss. & $\mathrm{x}$ & - \\
Moraceae & $\mathrm{x}$ & $\mathrm{x}$ \\
Sorocea bomplandii (Baill.) W. C.Burger et al. & & \\
\hline
\end{tabular}

Continua..
Tabela 1. continuação.

\begin{tabular}{|c|c|c|}
\hline Famílias/Espécie & $\mathrm{s} / \mathrm{g}$ & $\mathrm{c} / \mathrm{g}$ \\
\hline \multicolumn{3}{|l|}{ Myrtaceae } \\
\hline Blepharocalyx salicifolius (Kunth) O. Berg & $\mathrm{x}$ & - \\
\hline Eugenia uniflora $\mathrm{L}$. & $\mathrm{x}$ & - \\
\hline Eugenia uruguayensis Cambess. & $\mathrm{x}$ & - \\
\hline Myrcia glabra (O. Berg) D. Legrand & $\mathrm{x}$ & - \\
\hline Myrcia palustris DC. & $\mathrm{x}$ & - \\
\hline Myrcianthes gigantea (D. Legrand) D. Legrand & $\mathrm{x}$ & - \\
\hline Myrrhinium atropurpureum Schott & $\mathrm{x}$ & - \\
\hline \multicolumn{3}{|l|}{ Nyctaginaceae } \\
\hline Guapira opposita (Vell.) Reitz & $\mathrm{x}$ & - \\
\hline \multicolumn{3}{|l|}{ Primulaceae } \\
\hline Myrsine umbellata Mart. & $\mathrm{x}$ & - \\
\hline \multicolumn{3}{|l|}{ Rubiaceae } \\
\hline Psychotria brachyceras Müll.Arg. & $\mathrm{x}$ & $\mathrm{x}$ \\
\hline Psychotria carthagenensis Jacq. & $\mathrm{x}$ & $\mathrm{x}$ \\
\hline Psychotria leiocarpa Cham. \& Schltdl. & $\mathrm{x}$ & $\mathrm{x}$ \\
\hline \multicolumn{3}{|l|}{ Salicaceae } \\
\hline Casearia decandra Jacq. & $\mathrm{x}$ & - \\
\hline Xylosma pseudosalzmanii Sleumer & $\mathrm{x}$ & - \\
\hline \multicolumn{3}{|l|}{ Sapindaceae } \\
\hline Allophylus edulis (A. St.-Hill. et al.) Hieron. ex Niederl. & & $\mathrm{x}$ \\
\hline Cupania vernalis Cambess. & $\mathrm{x}$ & - \\
\hline \multicolumn{3}{|l|}{ Solanaceae } \\
\hline Solanum mauritianum Scop & $\mathrm{x}$ & $\mathrm{x}$ \\
\hline \multicolumn{3}{|l|}{ Thymelaeaceae } \\
\hline Daphnopsis racemosa Griseb & - & $\mathrm{x}$ \\
\hline
\end{tabular}

$\mathrm{s} / \mathrm{g}=$ parcelas sem a presença de gado e $\mathrm{c} / \mathrm{g}=$ parcelas com presença de gado. $\mathrm{x}=$ espécie presente, - = espécie ausente.

O maior número de indivíduos ocorreu na área sem gado (316) e em menor quantidade na área com gado (36). Essa diferença refletiu na densidade por área, equivalendo a 39.500 ind. ha ${ }^{-1}$ na área sem gado e 4.500 ind. ha $^{-1}$ na área com gado. A diferença estatística na riqueza e na densidade entre as duas áreas (Tabela 2), resultou em índice de diversidade de Shannon-Wiener mais elevado na área sem gado $\left(2,034\right.$ nats ind. $\left.{ }^{-1}\right)$ em relação à área com gado $\left(1,685\right.$ nats ind. $\left.{ }^{-1}\right)$. Isso demonstra que a área sem gado possui vegetação regenerante mais complexa, de maior diversidade e semelhante ao padrão de florestas tropicais. No entanto, o índice de equabilidade de Pielou foi 0,649 para a área sem gado e 0,732 para a área com gado. Esses valores mostram que as duas áreas apresentam baixa equabilidade, em virtude da dominância de poucas espécies na área sem gado, como as populações de Sorocea bonplandii, Trichilia elegans e o gênero Psychotria spp., que são espécies comuns e com altas densidades no sub-bosque das Matas de Restingas Arenosas no Rio Grande do Sul. Na área 
com gado, a baixa equabilidade é reflexo das poucas espécies tolerantes à pressão do pastoreio.

A estrutura da regeneração natural das duas áreas está representada nas Tabelas 3 e 4 . As espécies que apresentaram maior valor de importância na área sem gado foram $P$. carthaginensis $(25,53), S$. bonplandii $(24,62)$, P. brachyceras $(14,94), T$. elegans $(6,18)$ e Eugenia uruguayensis $(4,43)$. Na área com gado, Daphnopsis racemosa (50,86), P. brachyceras $(12,21)$,
Solanum mauritianum $(10,43)$ e $P$. carthaginensis $(9,02)$ foram as espécies predominantes.

Ocorreram seis espécies comuns às duas áreas, gênero Psychotria e as espécies T. elegans, Sebastiania brasiliensis e $S$. mauritianum. Na área com gado foram exclusivas D. racemosa, Ilex dumosa, Erythroxylum argentinum e Allophylus edulis. Outras 17 espécies foram exclusivas da área sem pecuária, com relevância para a família Myrtaceae.

Tabela 2. Valores de riqueza e de densidade nas áreas sem e com gado de Mata de Restinga Arenosa, Pelotas, extremo sul do Brasil.

\begin{tabular}{ccccccccc}
\hline & \multicolumn{4}{c}{ Sem gado } & \multicolumn{3}{c}{ Com gado } \\
& N & média & mínimo & máximo & N & média & mínimo & máximo \\
\hline Riqueza & $23 \mathrm{a}$ & 4,95 & 1 & 8 & $10 \mathrm{~b}$ & 1,3 & 0 & 4 \\
Densidade & $316 \mathrm{a}$ & 15,8 & 2 & 35 & $36 \mathrm{~b}$ & 1,8 & 0 & 5 \\
\hline
\end{tabular}

Letras distintas indicam diferenças significativas entre as médias dos índices analisados (teste $t ; p>$ 0,05). $\mathrm{N}=$ número total dos parâmetros.

Tabela 3. Parâmetros fitossociológicos da regeneração natural de uma Mata de Restinga sem pastoreio de gado, no extremo sul do Brasil.

\begin{tabular}{|c|c|c|c|c|c|c|}
\hline Espécies & $\mathbf{N}$ & DenRel & FreRel & DoRel & DoAbs & $\% \mathrm{VI}$ \\
\hline Psychotria carthaginensis & 91 & 28,8 & 80 & 33,46 & 2,5 & 25,53 \\
\hline Sorocea bonplandii & 82 & 25,95 & 85 & 28,91 & 2,16 & 24,62 \\
\hline Psychotria brachyceras & 62 & 19,62 & 65 & 12,08 & 0,9 & 14,94 \\
\hline Trichilia elegans & 15 & 4,75 & 35 & 6,71 & 0,5 & 6,18 \\
\hline Eugenia uruguayensis & 11 & 3,48 & 35 & 3,05 & 0,23 & 4,43 \\
\hline Psychotria leiocarpa & 10 & 3,16 & 35 & 1,87 & 0,14 & 4,14 \\
\hline Xylosma pseudosalzmanii & 5 & 1,58 & 25 & 2,98 & 0,22 & 3,21 \\
\hline Cupania vernalis & 9 & 2,85 & 15 & 4,33 & 0,32 & 2,75 \\
\hline Trichilia clausseni & 5 & 1,58 & 20 & 3,29 & 0,25 & 2,53 \\
\hline Sebastiania brasiliensis & 4 & 1,27 & 15 & 1,51 & 0,11 & 2,46 \\
\hline Sebastiania commersoniana & 6 & 1,9 & 10 & 0,31 & 0,02 & 1,98 \\
\hline Ocotea pulchella & 3 & 0,95 & 10 & 0,41 & 0,03 & 1,13 \\
\hline Guapira opposita & 2 & 0,63 & 10 & 0,17 & 0,01 & 0,94 \\
\hline Myrcianthes gigantea & 2 & 0,63 & 10 & 0,11 & 0,01 & 0,92 \\
\hline Myrcia palustris & 1 & 0,32 & 5 & 0,19 & 0,01 & 0,50 \\
\hline Solanum mauritianum & 1 & 0,32 & 5 & 0,16 & 0,01 & 0,50 \\
\hline Casearia decandra & 1 & 0,32 & 5 & 0,16 & 0,01 & 0,50 \\
\hline Blepharocalyx salicifolius & 1 & 0,32 & 5 & 0,13 & 0,01 & 0,49 \\
\hline Eugenia uniflora & 1 & 0,32 & 5 & 0,06 & $<0,01$ & 0,46 \\
\hline Myrrhinium atropurpureum & 1 & 0,32 & 5 & 0,05 & $<0,01$ & 0,46 \\
\hline Nectandra megapotamica & 1 & 0,32 & 5 & 0,02 & $<0,01$ & 0,45 \\
\hline Myrsine umbellata & 1 & 0,32 & 5 & 0,01 & $<0,01$ & 0,45 \\
\hline Myrcia glabra & 1 & 0,32 & 5 & 0,01 & $<0,01$ & 0,45 \\
\hline
\end{tabular}

$\mathrm{N}$ - número de indivíduos; DenRel - densidade relativa; FreRel - frequência relativa; DoRel - dominância relativa; DoAbs - dominância absoluta; VI - valor de importância (\%). 
Tabela 4. Parâmetros fitossociológicos da regeneração natural de uma Mata de Restinga usada como pastagem, no extremo sul do Brasil.

\begin{tabular}{lcccccc}
\hline \multicolumn{1}{c}{ Espécies } & N & DenRel & FreRel & DoRel & DoAbs & \%VI \\
\hline Daphnopsis racemosa & 17 & 47,22 & 55 & 64,63 & 0,32 & 50,86 \\
Psychotria brachyceras & 6 & 16,67 & 20 & 5,16 & 0,03 & 12,21 \\
Solanum mauritianum & 5 & 13,89 & 20 & 2,57 & 0,01 & 10,43 \\
Psychotria carthaginensis & 2 & 5,56 & 10 & 20,57 & 0,10 & 9,02 \\
Trichilia elegans & 1 & 2,78 & 5 & 1,61 & 0,01 & 4,86 \\
Psychotria leiocarpa & 1 & 2,78 & 5 & 3,06 & 0,02 & 3,18 \\
Erythroxylum argentinum & 1 & 2,78 & 5 & 0,93 & $<0,01$ & 2,47 \\
Sebastiania brasiliensis & 1 & 2,78 & 5 & 0,73 & $<0,01$ & 2,40 \\
Ilex dumosa & 1 & 2,78 & 5 & 0,45 & $<0,01$ & 2,31 \\
Allophylus edulis & 1 & 2,78 & 5 & 0,30 & $<0,01$ & 2,26 \\
\hline -
\end{tabular}

$\mathrm{N}$ - número de indivíduos; DenRel - densidade relativa; FreRel - frequência relativa; DoRel - dominância relativa; DoAbs - dominância absoluta; VI - valor de importância (\%).

As principais espécies da vegetação são diferentes entre as áreas. Exceção foi $P$. carthaginensis e $P$. brachyceras com abundância menor na área de pastoreio. Na floresta com gado habitam espécies comuns de estágios iniciais da sucessão ecológica, como D. racemosa, S. mauritianum e E. argentinum. $S$. mauritianum é uma planta ruderal, sendo encontrada facilmente em clareiras e bordas de fragmentos e terrenos baldios na área urbana. D. racemosa é planta comum em pastagens, e por vezes torna-se indesejada pela grande abundância nas áreas de pastoreio. Provavelmente, a espécie se mantém nas áreas pastoreadas porque traz na entrecasca substâncias tóxicas (Lombardo, 1964) e também cáusticas tanto para humanos quanto para o gado (Nevling Junior \& Reitz, 2008). Outro fator é a resistência das fibras da casca que podem ser usadas para fabricar cordas caseiras (Nevling Junior \& Reitz, 2008; Carrere, 2009) e que dão resistência e flexibilidade aos ramos e aos caules. Provavelmente, as toxinas e a resistência da casca são condicionadores do sucesso reprodutivo de D. racemosa nas áreas de pastoreio, pois os animais evitam a espécie como alimento, garantindo sua sobrevivência nas áreas pastoreadas.

$\mathrm{Na}$ floresta cercada foram encontradas populações semelhantes à estrutura original da floresta na região. Essas espécies são importantes para a composição futura da floresta. Destacam-se os gêneros Psychotria, Trichilia e a espécie $S$. bonplandii, plantas típicas do sub-bosque florestal e que habitam áreas de luz difusa no solo florestal. Também, plântulas adaptadas ao sombreamento das famílias Myrtaceae e Sapindaceae, estavam ausentes na área com gado.
No estudo da Mata do Totó, a área com pressão de pastoreio apresentou parâmetros reduzidos de riqueza, densidade, área basal e índice de diversidade. A alteração na estrutura natural da vegetação também foi encontrada sobre a influência do pastoreio em outros estudos. Santos \& Souza (2007) com Floresta Ripícola Estacional Semidecidual no estado do Rio Grande do Sul, encontraram falha no recrutamento de indivíduos jovens nas áreas pastejadas, sugerindo uma interrupção do processo de regeneração natural da população de Syagrus romanzoffiana (Cham.) Glassman, espécie de palmeira nativa na cidade de Pelotas.

Stern et al. (2002), em diferentes formações de Floresta Tropical na Costa Rica, notaram significativamente menos espécies nas áreas com pastoreio em comparação com a área sem pastejo. A estrutura da vegetação também foi diferente entre as áreas, sendo a área sem pastejo semelhante à floresta primária. Sampaio \& Guarino (2007) encontraram em fragmentos de Floresta Ombrófila Mista plântulas de espécies arbóreas com cobertura do solo significativamente menor nos sítios de alta intensidade de pastoreio. Cabe salientar, que Araújo (2010) observou que dois anos sem a presença de pastejo não foi suficiente para melhorar a regeneração natural de mata ciliar no município de Sant'ana do Livramento, RS.

Esses impactos ambientais na estrutura e na florística da floresta são derivados do pastoreio e do pisoteio dos animais, que afeta e elimina plântulas e espécies eretas de baixo porte (Vargas et al., 2002). O pisoteio e o pastoreio conduzem a regeneração natural para uma composição de espécies tolerantes à pressão do gado, como plantas espinhosas, com ramos e caules flexíveis 
e com substâncias de baixa palatabilidade aos animais. Isso promove simplificação da vegetação, fato que deve ser evitado no manejo sustentável de áreas de pastagem. Conforme FAO (2013) entre boas práticas na pecuária estão medidas de manejo do rebanho que minimizem impactos ambientais negativos ao meio ambiente. Para isso, é recomendada a presença de cercas para isolar as áreas, sejam áreas de preservação permanente, reserva legal ou destinadas na propriedade ao equilíbrio da paisagem, conforme o Código Florestal Brasileiro (Brasil, 2012). Katsuóka (2001) cita ainda áreas especiais de proteção do território, com restrições total ou parcial de uso: áreas marginais aos recursos hídricos, área de recarga de aquífero, encostas, áreas alagáveis ou de amortecimento de cheias, caminhos naturais de escoamento de águas, estuários, banhados e dunas.

\section{Conclusões}

A atividade pecuária alterou a estrutura da regeneração natural da floresta com presença de gado. Essas diferenças na vegetação são relacionadas à pecuária e secundariamente por diferentes níveis de abertura do dossel, proporcionado pela ausência de indivíduos regenerantes para recompor a floresta. Os dois trechos, mesmo que distantes $5 \mathrm{~m}$ entre si, apresentam diferenças significativas em relação à riqueza, densidade, diversidade e composição das principais famílias e espécies arbustivo-arbóreas da regeneração natural. Espécies ruderais e/ou com substâncias tóxicas e cáusticas, como Daphnopsis racemosa, foram beneficiadas na área com gado, enquanto espécies da família Myrtaceae e plantas esciófitas de sub-bosque foram prejudicadas.

No caso da Mata do Totó, que é um ambiente frágil e afetado por outras atividades antrópicas, o gado pode contribuir ainda mais para a degradação ambiental. Assim, é importante para a conservação das florestas que ocorra planejamento da atividade pecuária, uma vez que, o gado impede a regeneração natural adequada. Desde modo, recomenda-se o cercamento para promover o isolamento de remanescentes de vegetação natural em pastagens, técnica de recuperação de áreas degradadas para a conservação da vegetação na busca do equilíbrio da paisagem rural.

\section{Referências}

Alves, L. F. \& Metzger, J. P. A regeneração florestal em áreas de floresta secundária na Reserva Florestal do Morro Grande, Cotia, SP. Biota Neotropical, v. 6, n. 2, p. 1-26, 2006. DOI: 10.1590/ S1676-06032006000200005.

Araújo, A. C. B. Efeito do pastoreio de bovinos sobre a estrutura da mata ciliar do Arroio Espinilho em Sant'ana do Livramento, RS. 2010. 93 f. Dissertação (Mestrado em Engenharia Florestal) Universidade Federal de Santa Maria, Santa Maria, RS.

Brasil. Lei n ${ }^{\circ} 12.651$, de 25 de maio de 2012. Dispõe sobre a proteção da vegetação nativa e dá outras providências. Diário Oficial [da] República Federativa do Brasil, Brasília, DF, n. 102, p. 01-11, 25 maio 2012

Cândido, F. R. \& Silva, S. M. Impactos ambientais da pecuária leiteira em pequenas e médias propriedades rurais do Município de Barroso, MG. Revista Symposium, v. 8, n. 2, p. 109-126, 2010.

Carrere, R. La Envira (Daphnopsis racemosa): pionera, nodriza y testigo. 2009. Disponível em: < http://www.guayubira.org.uy/monte/ Envira.pdf>. Acesso em: 15 maio 2015.

Carvalho, J. O. P. Manejo de regeneração natural de espécies florestais. Belém: EMBRAPA-CPATU, 1984. 24 p. (EMBRAPACPATU. Documentos, 34).

Castilhos, Z. M. S. \& Pillar, V. P. Respostas ao pastejo ou exclusão podem ser caracterizados por tipos funcionais? Ciência Rural, v. 44, n. 1, p. 117-122, 2014. DOI: 10.1590/S0103-84782014000100019.

Dillenburg, L. R. et al. Species composition and structure of a sandy coastal plain forest in northem Rio Grande do Sul, Brazil. In: Seeliger, U. (Ed.). Coastal plant communities of Latin America. San Diego: Academic Press, 1992. p. 349-366.

Dorneles, L. P. P. \& Waechter, J. L. Estrutura do componente arbóreo da floresta arenosa de restinga do Parque Nacional da Lagoa do Peixe, Rio Grande do Sul. Hoehnea, v. 31, n. 1, p. 61-71, 2004.

Embrapa Clima Temperado. Laboratório de Agrometeorologia. Temperatura média $\left({ }^{\circ} \mathrm{C}\right)$ e precipitação pluviométrica $(\mathrm{mm})$ período: 1971/2000 (mensal/anual). 2011. Disponível em: $<$ http:// agromet.cpact.embrapa.br/estacao/mensal.html>. Acesso em: 29 dez. 2015

FAO. Guia de boas práticas na pecuária de leite. Roma, 2013. 41 p.

Gama, J. R. V. et al. Composição florística e estrutura da regeneração natural de floresta secundária de várzea baixa no estuário amazônico. Revista Árvore, v. 26, n. 5, p. 559-566, 2002. DOI: 10.1590/S010067622002000500005 .

IBGE. Vegetação: as regiões fitoecológicas, sua natureza e seus recursos econômicos: estudo fitogeográfico. In:

Levantamento de recursos naturais. Rio de Janeiro, 1986. v. 33. p. $541-632$.

Katsuóka, L. Avaliação do impacto da atividade agropecuária na qualidade da água em áreas de captação superficial nas bacias hidrográficas dos rios Mogi-Guaçu e Pardo, São Paulo. 2001. 223 f. Tese (Doutorado em Ciências da Tecnologia Nuclear) - Universidade Federal de São Paulo, São Paulo. 
Lombardo, A. Flora arborea y arborescente del Uruguai. Montevideo: Concejo Departamental de Montevideo, 1964. 151 p.

Lucas, R. W. et al. A. Riparian vegetation response to different intensities and seasons of grazing. Journal of Range Management, n. 57, n. 5 , p. $466-474,2004$. DOI: $10.2307 / 4003975$.

Magnago, L. F. S. et al. Os processos e estágios sucessionais da Floresta Atlântica como referência para a restauração florestal In: Martins, S. V. (Ed.). A Restauração Ecológica de Ecossistemas Degradados. Viçosa, MG: Ed. da UFV, 2012. p. 69-100.

Meers, T. L. et al. Role of plant functional traits in determining vegetation composition of abandoned grazing land in north-eastern Victoria, Australia. Journal of Vegetation Science, v. 19, n. 2, p. 515-524, 2008. DOI: 10.3170/2008-8-18401.

Moraes, D. \& Mondin, C. A. Florística e fitossociológia do estrato arbóreo em mata arenosa no balneário do Quintão, Palmares do Sul, Rio Grande do Sul. Pesquisas, Botânica, n. 51, p. 87-100, 2001.

Mueller-Dombois, D. \& Ellenberg, H. Aims and methods of vegetation ecology. New York: Willey \& Sons, 1974. 547 p.

Nevling Junior; L. I. \& Reitz, R. Timeleáceas. Itajaí: Herbário Barbosa Rodrigues, 1968. 21 p. (Flora ilustrada catarinense).

Pelotas (Prefeitura Municipal). Lei $\mathbf{n}^{\mathbf{0}}$ 4336, 18 de dezembro de 1998. Declara de valor paisagístico e ecológico a Mata do Totó, localizada no Balneário do Laranjal e Barro Duro. 1998. Disponível em: <http://www.pelotas.rs.gov.br/interesse_legislacao/leis/antigo/ L1998/Lei_n_4.336.pdf>. Acesso em: 03 jul. 2014.

Rosolen, V. et al. A. Impactos da substituição da vegetação original do cerrado brasileiro em sistemas agrícolas: alteração do carbono orgânico do solo e $\delta^{13} \mathrm{C}$. Investigaciones Geográficas, n. 79, p. 39-47, 2012.

Sampaio, M. B. \& Guarino, E. S. G. Efeitos do pastoreio de bovinos na estrutura populacional de plantas em fragmentos de Floresta Ombrófila Mista. Revista Árvore, v. 31, n. 6, p. 1035-1046, 2007. DOI: 10.1590/S0100-67622007000600008.

Santos, S. F. \& Souza, A. F. Estrutura populacional de Syagrus romanzoffiana em uma Floresta Ripícula sujeita as pastejo pelo gado. Revista Brasileira de Biociências, v. 5, supl. 1, p. 591-593, 2007.

Scherer, A. et al. Florística e estrutura do componente arbóreo de matas de restinga arenosa no Parque Estadual de Itapuã, RS, Brasil. Acta Botanica Brasilica, São Paulo, v. 19, n. 4, p. 717-726, 2005. DOI: $10.1590 / \mathrm{S} 0102-33062005000400006$.

Scherer, A. et al. Regeneração arbórea num capão de restinga no Rio Grande do Sul, Brasil. Iheringia, Série Botânica, v. 62, n. 1-2, p. 89-98, 2007.
Schulz, T. T. \& Leininger, W. C. Differences in riparian vegetation structure between grazed areas and exclosures. Journal of Range Management, v. 43, n. 4, p. 295-299, 1990. DOI: 10.2307/3898918.

Shepherd, G. J. Fitopac 2.1: manual do usuário. Campinas: Unicamp, 1995. $17 \mathrm{p}$.

Smale, M. C. et al. Long-term impacts of grazing on indigenous forest remnants on North Island hill country, New Zealand. New Zealand Journal of Ecology, v. 32, n. 1, p. 57-66, 2008.

Sosinski Júnior, E. E. \& Pillar, V. P. Respostas de tipos funcionais de plantas à intensidade de pastejo em vegetação campestre. Pesquisa Agropecuária Brasileira, v. 39, n. 1, p. 1-9, 2004. DOI: 10.1590/ S0100-204X2004000100001.

Stern, M. et al. Changes in composition and structure of a Tropical Dry Florest following intermittent cattle grazing. Revista Biologia Tropical, v. 50, n. 3/4, p. 1021-1034, 2002.

Szaro, R. C. \& Pase, C. P. Short-term changes in a Cottonwood-AshWillow association on a grazed and na ungrazed portion of Little Ash Creek in Central Arizona. Journal of Range Management, v. 38, n. 3, p. 382-384, 1983. DOI: 10.2307/3898493.

Tobler, M. W. et al. The impact of cattle ranching on large-scale vegetation patterns in a coastal savanna in Tanzania. Journal of Applied Ecology, v. 40, p. 430-444, 2003. DOI: 10.1046/j.13652664.2003.00816.x.

Universidade Católica de Pelotas. Relatório de pesquisa: curso de bacharelado em Ecologia. Pelotas, Laboratório de Geoprocessamento/ UCPEL, 2006.

Vargas, O. et al. Efecto del pastoreo sobre la estructura de la vegetación em un paramo humedo de Colombia. Ecotropicos, v. 15, n. 1, p. 35-50, 2002.

Venzke, T. S. et al. Florística e análise de similaridade de espécies arbóreas da mata da Praia do Totó, Pelotas, RS, Brasil. Ciência Florestal, v. 22, n. 4, p. 655-668, 2012. DOI: 10.5902/198050987548.

Venzke, T. S. Florística de comunidades arbóreas no Município de Pelotas, Rio Grande do Sul. Rodriguesia, v. 63, n. 3, p. 571-578, 2012. DOI: 10.1590/S2175-78602012000300008.

Waechter, J. L. Comunidades vegetais das restingas do Rio Grande do Sul. In: SIMPÓSIO DE ECOSSISTEMAS DA COSTA SUL E SUDESTE BRASILEIRA, 2., 1990, São Paulo. Anais... São Paulo: Academia de Ciência do Estado de São Paulo, 1990. p. 228-248.

Waechter, J. L. et al. Estrutura do componente arbóreo em uma floresta subtropical de planície costeira interna. In: SIMPÓSIO DE ECOSSISTEMAS BRASILEIROS, 5., 2000, Vitória. Anais... São Paulo: Academia de Ciência do Estado de São Paulo, 2000. p. 92-112. 
